\title{
Preparation and Electromagnetic Properties of Ni-Co Coated W-type Barium Ferrite Hollow Microspheres
}

\author{
Zhiguang $\mathrm{Li}^{1, \mathrm{a}}$, Jianjiang Wang ${ }^{1, \mathrm{a}}$, Weijuan $\mathrm{Mi}^{2, \mathrm{a}}$ and Liang $\mathrm{Yu}^{1, \mathrm{a}}$ \\ ${ }^{1}$ Mechanical Engineering College, Shijiazhuang 050003, China \\ ${ }^{2}$ Hebei Institute of Communication, Shijiazhuang 050051, china \\ aemail:Izgmwj@sohu.com
}

\begin{abstract}
Keywords: hollow microspheres; W-type barium ferrite; Ni-Co electroless plating; electromagntic properties

Abstract: The Ni-Co coated W-type barium ferrite $\left(\mathrm{BaZn}_{0.6} \mathrm{Co}_{1.4} \mathrm{Fe}_{16} \mathrm{O}_{27}\right)$ hollow microspheres (W-BFHMs) have been prepared by the self-reaction quenching technology and heat treatment at $1250{ }^{\circ} \mathrm{C}$. The W-BFHMs is a type of light microwave absorbing material in $\mathrm{GHz}$ frequencies and its average particle size is $60 \mu \mathrm{m}$. A conductive and magnetic Ni-Co layer was coated on the surface of W-BFHMs by electroless plating. Effects of plating time on the particle size, morphology, phase structure and microwave absorption properties of Ni-Co coated W-BFHMs are investigated through SEM, XRD, particle size analyzer and vector network analyzer. The results show that the Ni-Co deposits in the sag and the trench firstly and grows as the branch after filling the sag and the trench. With the increase of the plating time from $5 \mathrm{~min}$ to $25 \mathrm{~min}$, the magnetic loss increase from 0.15 to 0.35 , while the dielectric loss is not significantly change due to the incompletely covered. The effective absorbing bandwidth increase from $1 \mathrm{GHz}$ to $2.7 \mathrm{GHz}$, while the absorption peak decrease from $-12 \mathrm{~dB}$ to $-32 \mathrm{~dB}$ when the plating time is $25 \mathrm{~min}$.
\end{abstract}

\section{Introduction}

The development of the radar stealth technology and the target shape technology are more and more limited to the tactical index. Application ranges have been limited due to shortcomings of bandwidth, low efficiency and large density. It is urgent need to develop new high performance light microwave absorbing materials [1-2].

Hollow microsphere materials are the important direction of the light microwave absorbing materials. At present, more research is concentrated on the electromagnetic absorption material of coating layer (such as $\mathrm{Ni} 、 \mathrm{Co} 、 \mathrm{Fe}-\mathrm{Ni} 、 \mathrm{Fe}-\mathrm{Co}$ ) $[3,4,5]$. However, the basic materials are given priority to the fly ash microspheres or hollow glass microspheres, which do not have the microwave absorbing properties. Zhao Yan [6] plated Co on the surface of hollow microspheres and analysed the electromagnetic properties in $2 \sim 18 \mathrm{GHz}$. Sung-Soo Kim [7] plated a layer of Co-Fe alloy thin films on hollow ceramic microspheres. High absorption rate and thin matching thickness are predicted in the composite layers. K. N. Ge [8] plated Ni on the surface of the fly ash microsphere by the method of electroless plating. The effective frequency bandwidth and absorption peak are $16.6 \sim 18 \mathrm{GHz}$ and $-13.57 \mathrm{~dB}$. Although these methods of compound of the microwave absorbing materials decrease the weight, but the loss mechanism of one layer electromagnetic absorption material is single and the impedance matching characteristics is relatively poor. It is difficult to meet the requirements "thin, light, wide and strong" of new microwave absorbing materials.

The W-BFHMs have been prepared by self-reaction quenching technology (SRQT) and heat treatment. W-BFHMs have the advantages of low density, high temperature resistant, corrosion resistance, etc. On the other hand, W-BFHMs have electromagnetic absorption performance. Therefore, W-BFHMs are taken as the base material. The Ni-Co layers on the surface are plated by electroless plating. The change of the microstructure and dual loss mechanism of double-layer hollow microspheres have been studied under different ultrasonic electroless plating time. 


\section{Experiment}

\subsection{Preparation of $\mathrm{W}-\mathrm{BFHMs}$ principle and process}

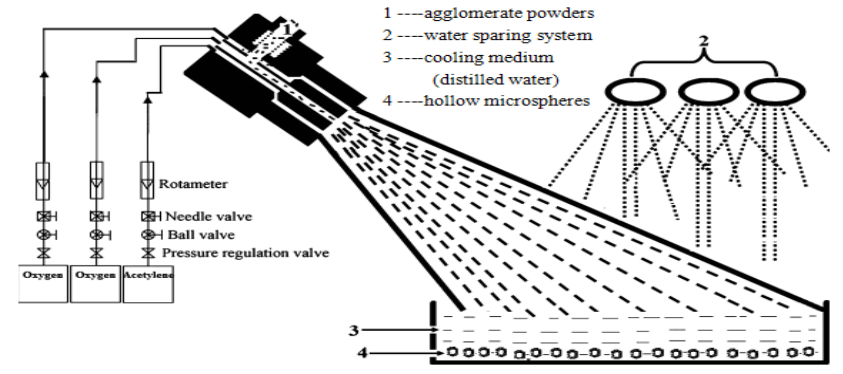

Fig.1 Principle diagram for preparation of hollow microspheres using SRQT

The preparation principle diagram of hollow microspheres has been showed in Fig. 1. The agglomerate powders are sprayed into the flame filed through a CP-D ${ }_{I I I}$-type high-energy flame-spraying gun. When reaching the ignition temperature, the self-propagation high-temperature synthesis (SHS) reaction occurs promptly and the temperature of reaction system exceeds the melt point of products. Simultaneously, large volume of gas is produced which results in the hollow structure of drops. Then, the drops are quenched quickly into the cooling medium (the distilled water). Because the gas cannot escape from the drops, hollow microspheres are obtained immediately after drying and filtering the quenching products. Put the hollow microspheres into the tubular electric furnace heating to $1250{ }^{\circ} \mathrm{C}$ at a rate of $5^{\circ} \mathrm{C} / \mathrm{min}$. After five hours heat treatment, the products of W-BFHMs have been prepared.

The reaction equations is showed in Eq. (1) and (2).

$3 \mathrm{Fe}+3 \mathrm{Fe}_{2} \mathrm{O}_{3}+\mathrm{BaCO}_{3}+\mathrm{ZnCO}_{3}+\mathrm{CoCO}_{3}+\mathrm{KClO}_{4} \rightarrow$

$$
\mathrm{ZnFe}_{2} \mathrm{O}_{4}+\mathrm{BaFe}_{2} \mathrm{O}_{4}+\mathrm{CoFe}_{2} \mathrm{O}_{4}+\mathrm{Fe}_{3} \mathrm{O}_{4}+3 \mathrm{CO}_{2}+\mathrm{KCl}
$$

$5 \mathrm{Fe}_{2} \mathrm{O}_{3}+0.6 \mathrm{ZnFe}_{2} \mathrm{O}_{4}+1.4 \mathrm{CoFe}_{2} \mathrm{O}_{4}+\mathrm{BaFe}_{2} \mathrm{O}_{4} \rightarrow \mathrm{BaZn}_{0.6} \mathrm{Co}_{1.4} \mathrm{Fe}_{16} \mathrm{O}_{27}$

According to the molecular formula, the mole ratio of Eq. (1) and (2) have been determined. The analytical reagent raw materials of Fe powders, $\mathrm{BaCO}_{3}, \mathrm{Fe}_{2} \mathrm{O}_{3}, \mathrm{CoCO}_{3}, \mathrm{ZnCO}_{3}$ and $\mathrm{KClO}_{4}$ were selected.

Firstly, the raw materials were put into the LJM-5L ball mill and anhydrous ethyl alcohol was taken as the medium sphere to be churned for $6 \mathrm{~h}$. After that, the epoxy resin was added into the mill to be stirred for another $2 \mathrm{~h}$. Epoxy resin was used to cooperate with sucrose and to enlarge the contact area of the components in the agglomerate powders. It can be transformed to $\mathrm{CO}, \mathrm{CO}_{2}$, and $\mathrm{H}_{2} \mathrm{O}$ in SHS reaction due to the high temperature. Secondly, the mixtures were dried and carbonized at $200^{\circ} \mathrm{C}$ until no smoke releases. The agglomerate powders were selected with $38 \sim 45 \mu \mathrm{m}$ for the experiment.

\subsection{Preparation of Ni-Co coated W-BFHMs}

The Ni-Co layers have been coated on the surface of W-BFHMs by the method of ultrasonic electroless plating. Ultrasonic electroless plating is a technology of preparing composite powder and can form a uniform layer of other metal materials [9,10]. Electroless plating is an electrochemical reaction. The reaction was conducted in alkaline conditions. $\mathrm{H}_{2} \mathrm{PO}_{2}^{-}$occur the oxidation reaction while $\mathrm{Ni}^{2+}$ and $\mathrm{Co}^{2+}$ occur the reduction reaction. The premise conditions of reaction is the material surface needs certain active center, to make the electrochemical reaction occurs. Therefore, pretreatment on the material is needed before processing. In addition, adding appropriate complexing agent can prevent the formation of $\mathrm{Ni}$ and $\mathrm{Co}$ hydroxide precipitation before ultrasonic electroless plating.

The pretreatment before plating is composed of four steps of roughening (30 min), sensitization(15 $\mathrm{min})$, activation $(10 \mathrm{~min})$ and reduction $(10 \mathrm{~min})$. The temperature of four steps is at room temperature. $2 \mathrm{~g}$ of the pretreatment W-BFHMs was placed in $500 \mathrm{~mL}$ plating solution which have $10 \mathrm{~g}$ 
$\mathrm{NiSO}_{4} \cdot 6 \mathrm{H}_{2} \mathrm{O}, 10 \mathrm{~g} \quad \mathrm{CoSO}_{4} \cdot 7 \mathrm{H}_{2} \mathrm{O}, \quad 8.5 \mathrm{~g} \quad \mathrm{NaPH}_{2} \mathrm{O}_{2} \cdot \mathrm{H}_{2} \mathrm{O}$ and $57.5 \mathrm{~g} \quad \mathrm{Na}_{3} \mathrm{C}_{6} \mathrm{H}_{5} \mathrm{O}_{7} \cdot 2 \mathrm{H}_{2} \mathrm{O}$ at $80{ }^{\circ} \mathrm{C}, \mathrm{pH}=9.0$.

2.3 Characterization and detection methods. The morphology of the W-BFHMs and Ni-Co coated W-BFHMs were detected by scanning electronmicroscope (SEM, QUANTA FEG-250). The phase composition was studied by X-ray diffraction (XRD, BRUKER D2 PHASER). Particle size distribution was measured by Laser Particle Size Analyzer (Beckman Coulter LS 13 320, testing from $0.04 \mu \mathrm{m}$ to $2000 \mu \mathrm{m}$ ). The density of W-BFHMs was determined by Archimedes Method.

The microwave absorbing specimens were prepared by molding with paraffin. The mix weight ratio of Ni-Co coated $\mathrm{W}-\mathrm{BFHMs}$ and paraffin are 3:2. The specimens have a toroidal shape with the thickness at $2.0 \mathrm{~mm}$, and the outer and inner diameters are respectively $7.0 \mathrm{~mm}$ and $3.0 \mathrm{~mm}$. The $\varepsilon^{\prime}, \varepsilon^{\prime \prime}$, $\mu^{\prime}$ and $\mu^{\prime \prime}$ versus frequency were measured by coaxial reflection-transmission method with Vector Network Analyzer (Agilent-N5242A) in 0.5-18 GHz.

The microwave absorbing characteristics can be represented by the reflection loss (RL), as shown in Eq. (3) and (4). $Z_{i n}$ is the normalized input impedance related to the impedance in free space. The $\varepsilon_{r}$ is the complex relative permeability and the $\mu_{r}$ is permittivity of the material, $d$ is the thickness of the absorber, and the $c$ is the velocity of light and $f$ is the frequency of microwave.

The RL value is calculated by the metlab at the thickness $2.0 \mathrm{~mm}$ with the Eq. (3) and (4) $[11,12]$.

$$
\begin{aligned}
& \mathrm{RL}=20 \lg \left|\frac{Z_{\text {in }}-1}{Z_{\text {in }}+1}\right| \\
& Z_{\text {in }}=\left(\frac{\varepsilon_{\mathrm{r}}}{\mu_{\mathrm{r}}}\right)^{1 / 2} \tanh \left[j\left(\frac{2 \pi f d}{\mathrm{c}}\right)\left(\mu_{\mathrm{r}} \varepsilon_{\mathrm{r}}\right)^{1 / 2}\right]
\end{aligned}
$$

\section{Results and discussion}

3.1 Effect of the morphology and element at different electroless plating time. The SEM images of W-BFHMs and Ni-Co coated W-BFHMs at different ultrasonic electroless plating time have been shown in Fig. 2. Fig. 2(a) and 2(b) are W-BFHMs without electroless plating.
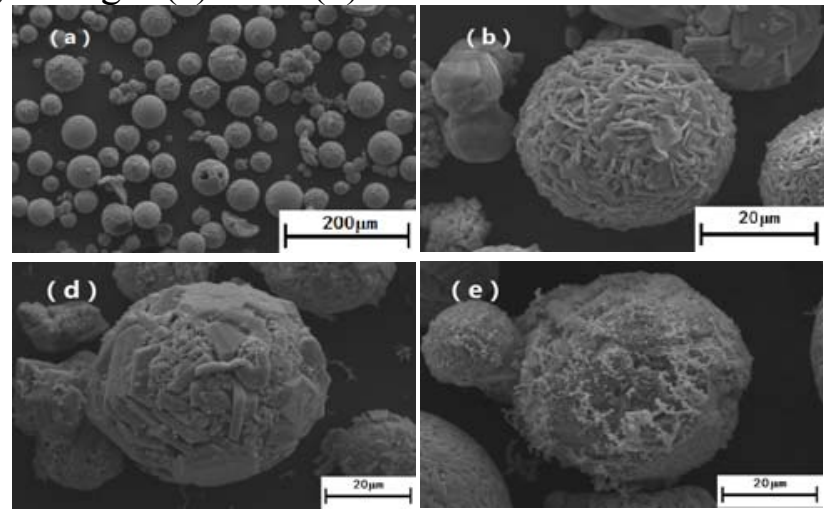

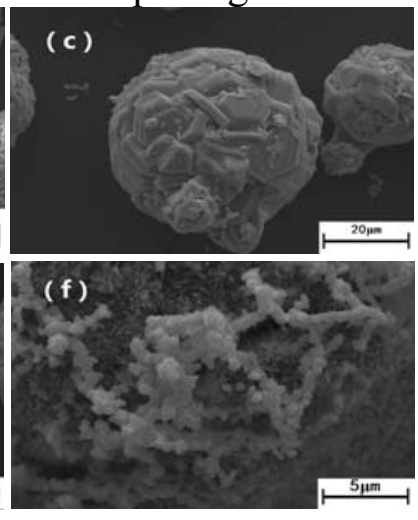

Fig. 2 SEM images of the W-BFHMs and the Ni-Co coated W-BFHMs

It can be seen that W-BFHMs are mainly composed of a large number of closed spherical particles and individual opening spherical particles. The average particle size is $60 \mu \mathrm{m}$. Fig. 2(c), 2(d) and 2(e) are plated with time $5 \mathrm{~min}, 15 \mathrm{~min}$ and $25 \mathrm{~min}$, respectively. It can be seen that at the early reaction on the surface of the W-BFHMs, tiny Ni-Co particles begin to appear. With the increase of reaction time, particle increased continuously. With these branches interlacing and stacking, the morphology is formed as shown in figure 2(f). It is mainly due to the lack of reaction time and the concentration of the roughening solution. This is not conducive to the adhesion and growth of nickel particles in the early of reaction and the rate of reaction is reduced. 


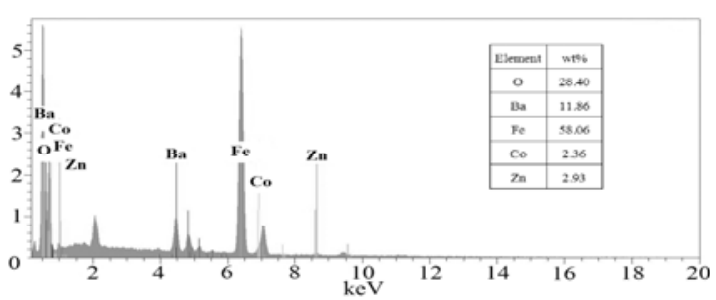

(a)

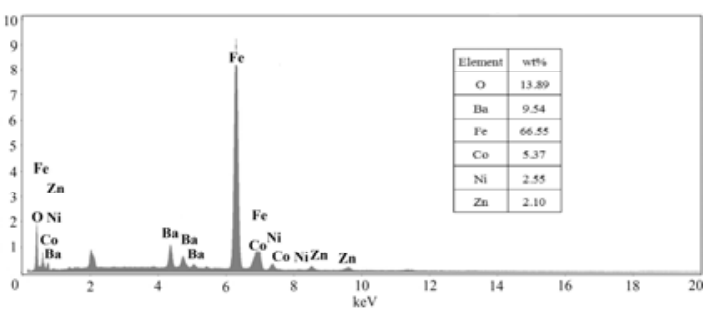

(b)

Fig.3 EDS patterns of the W-BFHMs and after ultrasonic electroless plating (5 min)

The energy spectrums of W-BFHMs and after ultrasonic electroless plating are analyzed in Fig. 3. The results show that the products consist of $\mathrm{Ba}, \mathrm{Fe}, \mathrm{Co}, \mathrm{Zn}$ and $\mathrm{O}$ elements before ultrasonic electroless plating(Fig. 3(a)), without Ni element. However, the product of Ni-Co element increase after ultrasonic electroless plating (Fig. 3(b)).

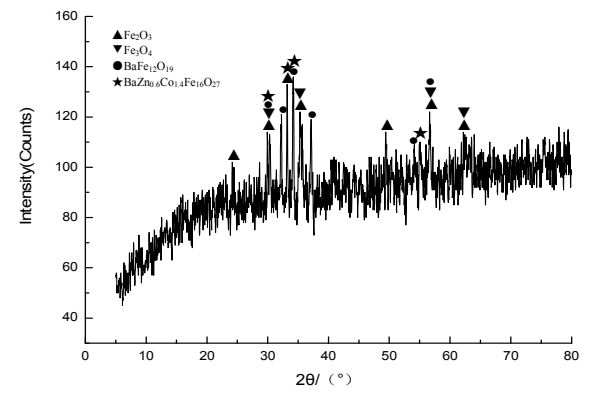

(a)

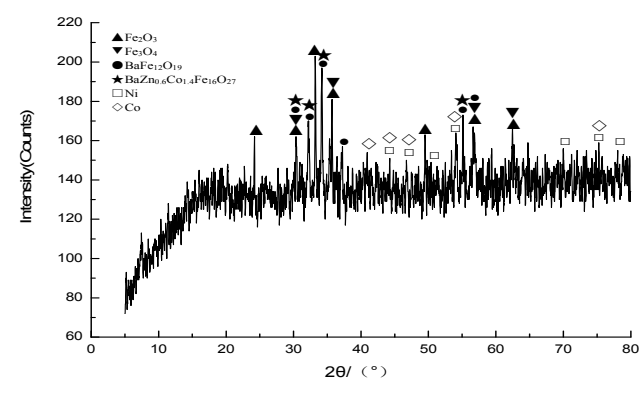

(b)

Fig. 4 XRD pattern of the W-BFHMs and after ultrasonic electroless plating (5 min)

The crystal type develops completely to hexagonal shape crystal structure $\left(\mathrm{BaZn}_{0.6} \mathrm{Co}_{1.4} \mathrm{Fe}_{16} \mathrm{O}_{27}\right)$ after heat treatment at $1250{ }^{\circ} \mathrm{C}$ which has been shown in Fig. 4(a). It is shown that the Ni-Co element increase after ultrasonic electroless plating in Fig. 4(b) .

\subsection{Effect of the electromagnetic properties at different electroless plating time}
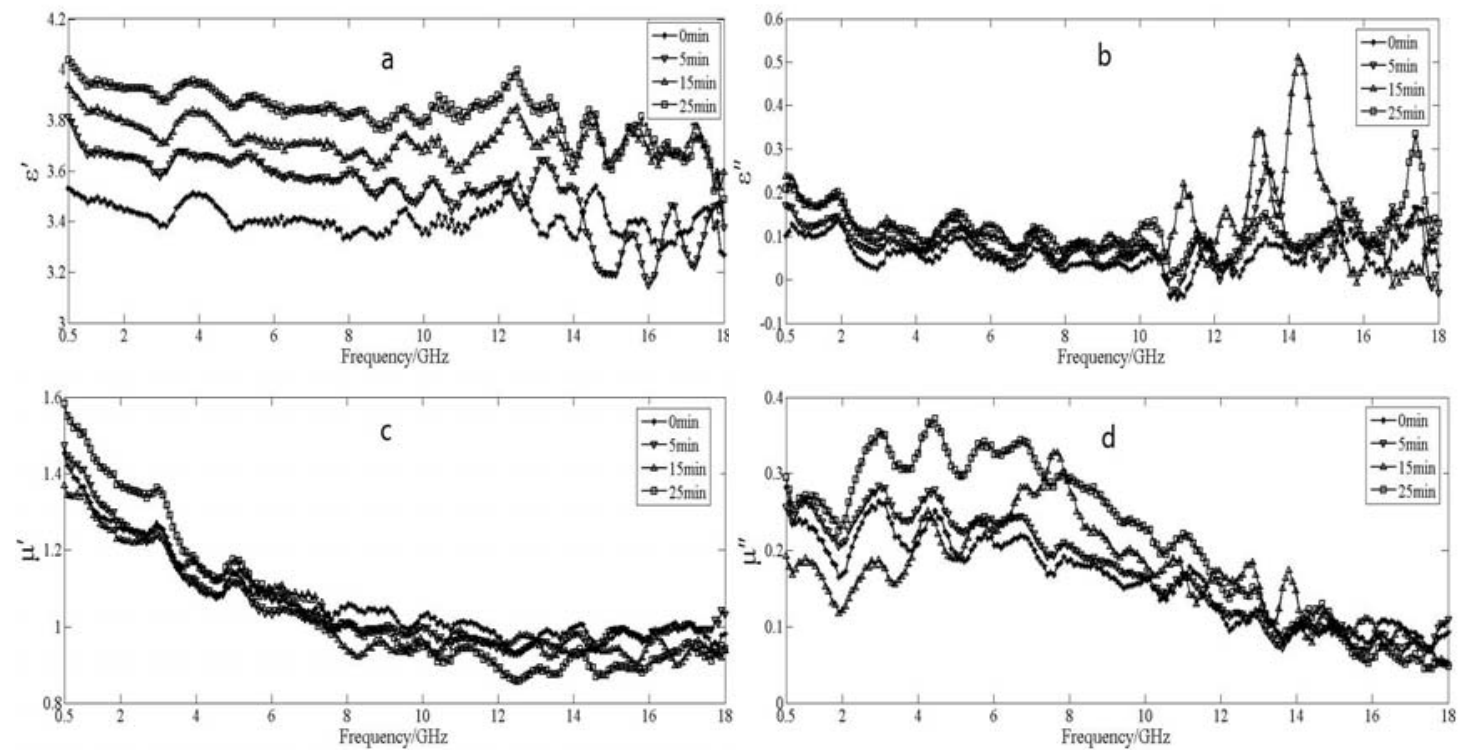

Fig. 5 Curves of electromagnetic parameters at different electroless plating time

Fig. 5 shows the curves of electromagnetic parameters $\left(\varepsilon^{\prime}, \varepsilon^{\prime \prime}, \mu^{\prime}\right.$ and $\left.\mu^{\prime \prime}\right)$ of the Ni-Co coated W-BFHMs at different electroless plating time (0,5 $\mathrm{min}, 15 \mathrm{~min}$ and $25 \mathrm{~min})$. 
The Figure 5(a) shows curves of electromagnetic parameters $\varepsilon^{\prime}$ of the W-BFHMs at different ultrasonic electroless plating time. It can be seen that with the increase of frequency, the real part of complex permittivity $\varepsilon^{\prime}$ remains generally around a value. But with the increase of plating time $\varepsilon^{\prime}$ increased from 3.4 to 4 . However, the imaginary parts of permittivity $\varepsilon^{\prime \prime}$ are not change significantly between $0.5 \sim 12 \mathrm{GHz}$, only a slight increase of peak between $10 \sim 16 \mathrm{GHz}$ as shown in figure $5(\mathrm{~b})$.

The figure 5(c) shows that the real parts of permeability $\mu$ ' reduce with the increase of frequency after $8 \mathrm{GHz}$ basically stable at 1 . With the increase of plating time, it increase before $6 \mathrm{GHz}$ and decreases after $6 \mathrm{GHz}$. The figure $5(\mathrm{~d})$ shows that the imaginary parts of permeability $\mu$ " significantly increase within $0 \sim 12 \mathrm{GHz}$ and the peak increases from 0.2 ( $0 \mathrm{~min})$ to 0.35 (25 $\mathrm{min})$.

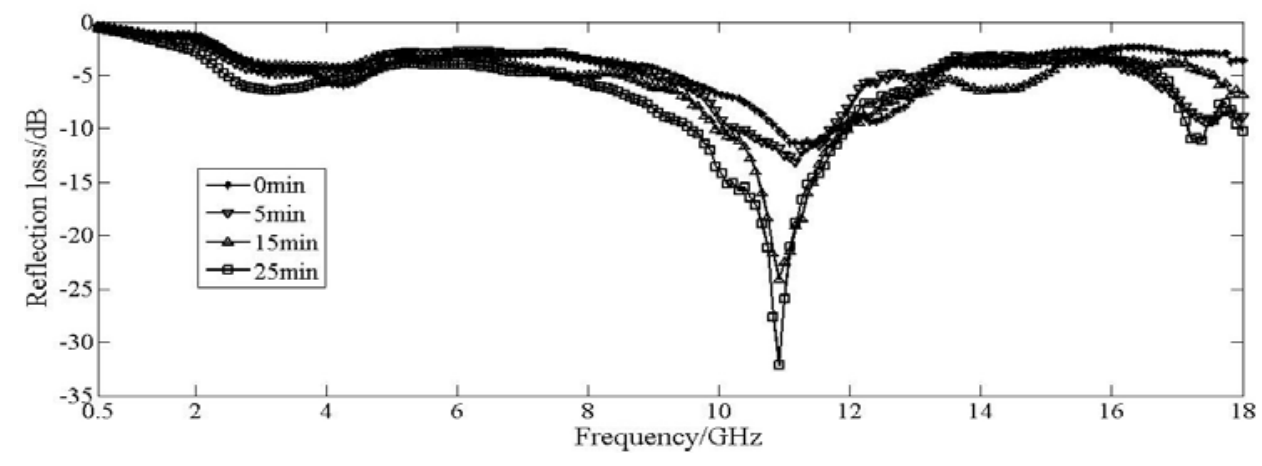

\section{Fig. 6 Curve of Reflection loss at different ultrasonic electroless plating time}

It is shown that the W-BFHMs have microwave absorption performance and its magnetic loss peak is at $12 \mathrm{GHz}$ within $0.5 \sim 18 \mathrm{GHz}$ in Fig. 6. In the same way, the loss mechanism of Ni-Co as the magnetic material is given priority to natural resonance. Therefore, when a certain amount of Ni-Co coated on the surface of W-BFHMs, on the basis of the original magnetic loss was further improved.

The Fig. 6 shows that effective absorbing bandwidth (less than $-10 \mathrm{~dB}$ ) of W-BFHMs is less than $1 \mathrm{GHz}$ whin $0.5 \sim 18 \mathrm{GHz}$ and the absorption peak is only at $-12 \mathrm{~dB}$. With the increase of plating time, it is shown that the Ni-Co coated W-BFHMs have stronger absorption capacity. When the plating time is $5 \mathrm{~min}$, its effective absorbing bandwidth is $10 \sim 11.8 \mathrm{GHz}$, and the absorption peak is $-14 \mathrm{~dB}$; When the plating time is $15 \mathrm{~min}$, its effective absorbing bandwidth is $9.9 \sim 12 \mathrm{GHz}$ and the absorption peak is $-24 \mathrm{~dB}$; When the plating time is $25 \mathrm{~min}$, the effective wave absorption bandwidth is $9.3 \sim 12$ $\mathrm{GHz}$ and the absorption peak is $-32 \mathrm{~dB}$. So, with the increase of Ni-Co coated plating time, its effective absorbing bandwidth become widely from $1 \mathrm{GHz}$ to $2.7 \mathrm{GHz}$ and absorption peaks increase from $-12 \mathrm{~dB}$ to $-32 \mathrm{~dB}$. At the same time, the microwave absorption performance also presents different degrees of enhancement within $2 \sim 8 \mathrm{GHz}$.

\section{Conclusions}

(1) By the self-reaction quenching technology, heat treatment and ultrasonic electroless plating, W-BFHMs and Ni-Co coated W-BFHMs have been prepared with the average particle size of $60 \mu \mathrm{m}$. The W-BFHMs are mainly composed of spherical hollow microspheres. Ni-Co deposits in the sag and trench of hexagonal lamellar crystal of W-BFHMs firstly, it grows like the branch after filling the sag and trench.

(2) At the different electroless plating time, the real part permittivity $\varepsilon^{\prime}$ increases from 3.4 to 4 and the imaginary part of permittivity $\varepsilon^{\prime \prime}$ does not change significantly between $0.5 \sim 12 \mathrm{GHz}$, only a slight increase of peak between $10 \sim 16 \mathrm{GHz}$; the real part of permeability $\mu^{\prime}$ slight increase before $6 \mathrm{GHz}$ (from 1.4 to 1.6), while decreased after $6 \mathrm{GHz}$ (from 1 to 0.9 ), the imaginary part of permeability $\mu^{\prime \prime}$ significantly increases within $0.5 \sim 12 \mathrm{GHz}$ and the peak increases from $0.2(0 \mathrm{~min})$ to 0.35 (25 $\mathrm{min})$. The change of electromagnetic loss mechanism of Ni-Co coated W-BFHMs mainly displays in the increase of magnetic loss. Its magnetic loss mainly comes from the natural resonance. 
(3)The microwave absorption performance of Ni-Co coated W-BFHMs at the plating time of 25 min has improved significantly. Its effective absorbing bandwidth is from $1 \mathrm{GHz}$ to $2.7 \mathrm{GHz}$ and absorption peaks increase from $-12 \mathrm{~dB}$ to $-32 \mathrm{~dB}$ with the increase of plating time. So it can be expected that strong dielectric loss and the obvious absorption peak appear in $12 \sim 16 \mathrm{GHz}$ when the W-BFHMs have been fully coated.

\section{Acknowledgements}

This work was financially supported by the National Natural Science Foundation (51172282).

\section{References}

[1] E. H. Ha, D.Q. Huang, H.Y. Ding, et al. Application research and prospects of new and light mass radar absorbing materials[J]. Materials Engineering, 2006, 3:55-59.

[2] M. J. Iqbal, R. A. Khan, S. Mizukami. Tailoring of structural, electrical and magnetic properties of $\mathrm{BaCo}_{2} \mathrm{~W}$-type hexaferrites by doping with $\mathrm{Zr}-\mathrm{Mn}$ binary mixtures for useful applications[J]. Journal of Magnetism and Magnetic Materials, 2011, 323:2137-2144.

[3] J. Xiang, X. H. Zhang, Q. Ye, et al. In-situ Preparation and Microwave Absorption Performances of Fe-Ni/C Composite Nanofibers[J]. Chemical Journal of Chinese Universities, 2014, 35(7): 1379-1387.

[4] L. Z. Xia, H. Z. Zheng, M. L.Xiao. Preparation and apllication in electromagnetic absorption of ZnO/FeNiMo composite[J]. Rare Metals, 2014, 33(5): 573-577.

[5] M.Yu, J. H. Liu, S. M. Li, et al. Fabrication of Ni coatings on glass microspheres and electromagnetic properties[J]. Materials Engineering, 2009, 6:1-4.

[6] Y. Zhao, T. Zhang, R. Zhang, et al. Hollow ceramic microsphere Coated with Co by electroless plating and its electromagnetic properties[J]. Rare Metal Materials and Engineering, 2010, 39(4): 0587-0592.

[7] S. S. Kim, S. T. Kim, J. M. Ahn, et al. Magnetic and microwave absorbing properties of Co-Fe thin films plated on hollow ceramic microspheres of low density[J]. Journal of Magnetism and Magnetic Materials, 2004, 271: 39-45.

[8] K. N. Ge, Q. Wang, Q. J. Mao, et al. Surface modification on cenosphere and its wave absorbing properties[J]. Journal of Functional Materials and Devices, 2003, 9(1):67-70.

[9] J. H. Kim, S. S. Kim. Microwave absorbing properties of Ag-coated Ni-Zn ferrite microspheres prepared by electroless plating[J]. Journal of Alloys and Compounds, 2011, 509:4399-4403.

[10]H. Y. Wang, C. X. Zhang, S. X. Yu, et al. Microwave Absorbing properties of the electroless nickel-phosphorus deposited on the hollow microspheres[J]. Surface technology, 2010, 39(6):63-74.

[11]J. B. Li, X. Wu, L. C. Li, et al. Preparation and Electromagnetic Properties of Zn-Ni-Cu ferrites/poly(O-methoxyaniline) composites[J]. Scientia Sinica Chimica, 2014, 44(3): 309-319.

[12]J. Xiang, X. H. Zhang, Q. Ye, et al. Structural design and absorption properties of double layer microwave absorbers based on $\mathrm{Li}_{0.35} \mathrm{Zn}_{0.3} \mathrm{Fe}_{2.35} \mathrm{O}_{4}$ and Carbon Nanofibers[J]. Chinese Journal of Inorganic Chemistry, 2014, 30(4): 845-852. 